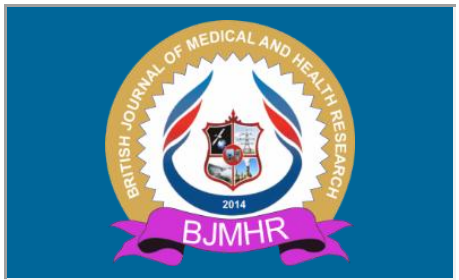

\title{
BJMHR
}

British Journal of Medical and Health Research Journal home page: www.bjmhr.com

\section{Effect of Dry Needling with K-Taping in Management of Chronic Back Pain- Case Report}

\author{
Abdullah S. Bin Hussein ${ }^{1}$, Faisal K. Alhabib ${ }^{2}$, Dr.Sharick Shamsi ${ }^{3}$ \\ 1.Deputy Director of Physiotherapy Dept., PSMMC, Riyadh, KSA, \\ 2.Physiotherapist at PSMMC, Riyadh KSA, \\ 3.Senior Physiotherapist at PSMMC, Riyadh KSA,
}

\section{ABSTRACT}

Myofascial trigger points (MFTP) are the first choice of treatment for all researchers and clinicians in neuro musculoskeletal pain. Trigger point dry needling (TPDN) is an invasive technique involving MFTP stimulation by monofilament needle. The aim of this case study is to find out results of TPDN and K-Taping as a main choice of treatment in chronic low back pain patient. A 35-year-old male patient working as senior physiotherapist, suffering from LBP since 2 years. He reveals history of lifting and transferring a heavy patient from chair to bed 2 years ago. The senior Therapist has been treating patients in outpatient orthopedic clinic for 10 years. Visual Analog Scale (VAS) score (where 0 indicates no pain and 10 maximum possible pain) was $6 / 10$ before intervention. The patient was treated for para spinal muscles with TPDN and K-Taping, with core muscle strengthening exercise protocol for 2 sessions with 3 days interval. There was no pain (0/10) reported on VAS Scale after intervention and no impairment or functional limitations, including normal range of motion in all direction and no MFTP on palpation. The patient was able to get back to work without any problem and he was able to resume his previous responsibilities without pain. TPDN with K-Tape intervention proved beneficial for the treatment of chronic low back pain. Further future research is recommended to know if TPDN intervention, with K-Tape is effective in other body parts pain and long-term treatment follow up in larger patient group.

Keywords: Dry needling, K-Taping, chronic low back pain, myofasical trigger point 


\section{INTRODUCTION}

Prevalence of low back pain estimated $23 \%$ at one point ${ }^{1},{ }^{2}$ low back pain (LBP) has been a major public health challenge in the world, contributing great medical burden. It is also becoming one of the leading specific causes of disability worldwide ${ }^{1-3}$. Low back pain (LBP) is a worldwide health problem and the most common reason for musculoskeletal disorders, especially in sedentary people, and even in highly trained athletes ${ }^{4,5}$. It has been estimated that as several as eighty fifth of voters in developed countries expertise LBP at some purpose throughout their lifetime; thus, LBP is one of the commonest cause of appointments to outpatient department and emergency centres ${ }^{5,6}$. LBP is commonly treated conservatively by physiotherapy, acupuncture, dry needling, massage and analgesics ${ }^{5,7,8}$. Pharmacological treatment remains a mainstream therapy for LBP but it is still not satisfactory, considering the potential harms caused by medication such as non-steroidal anti-inflammatory drugs and opioids in the management of $\mathrm{LBP}^{9}$.

Dry needling (DN), as a relatively new treatment modality practiced by physicians worldwide, is often used to treat musculoskeletal pain (including LBP) and has attracted more and more interest $^{2,10}$. It involves a minimally invasive procedure in which an acupuncture needle is inserted directly into myofascial trigger points ${ }^{2,11}$.Acupuncture needle is used, unlike conventional acupuncture that is based on the theory of traditional Chinese medicine, DN is developed along with the theory of MTrPs ${ }^{11}$. It is generally accepted that DN no longer involves traditional Chinese medicine concepts and belongs to a subcategory of western medical acupuncture ${ }^{10,12}$. In present time, an increasing number of trials have been performed to explore the efficacy of DN for treating pain; however, the conclusions were conflicting. The real effectiveness of DN remains controversial. So, it is urgent to seek for the evidence of this therapy for treating pain ${ }^{12}$.

Kinesio taping $(\mathrm{KT})$ is a conservative treatment method for controlling pain in the treatment of musculoskeletal conditions which have recently gained popularity. $\mathrm{K}$ tape technique has 2 concepts with varying tension during application. With light (15-25\%) tension application of method from insertion point to origin of the muscle, the muscular function is inhibited, whereas with light to moderate (25-50\%) tension application of the method from origin of muscle to insertion point activates the muscular Function ${ }^{13,14}$ K Taping technique helps to improve natural healing mechanism of body by supporting and stabilizing muscles and joints, with no limitation in range of motion. It can be applied to various muscular problems. Kenzo Kase, pioneered in developing $\mathrm{K}$ taping technique, based on special elastic strips, which has elasticity and density similar to our skin. It is not having any drug or chemical ,contains $100 \%$ cotton and is waterproof. It also improves lymphatic drainage ${ }^{15,16}$. K Taping Application results in 
skin interstitial space expansion and can be remain for 3-5 days or till any skin irritation or $\operatorname{rash}^{15,16}$. Physiotherapy treatment process requires application of manual therapy techniques, frequent transferring and lifting patients ${ }^{17,18}$ which may lead to work related musculoskeletal disorders (WMSD) in physiotherapists. Due to WMSD Physiotherapists remain absent (32\%) or leave their profession $(17-30 \%)^{19,20}$.

The research regarding effects of Dry needling along with K Taping on occupational LBP is lacking and is not enough, so we are describing a case of a physiotherapist with chronic LBP related to patient handling and the effect of Dry needling along with KT on of LBP treatment.

\section{CASE REPORT}

A 35-year-old male patient working as senior physiotherapist, suffering from LBP since 2 years. He reveals history of lifting and transferring a heavy patient from chair to bed 2 years ago. The senior Therapist has been treating patients in outpatient orthopedic clinic for 10 years. Visual Analog Scale (VAS) score (where 0 indicates no pain and 10 maximum possible pain) was $6 / 10$ before intervention. The patient was treated for para spinal muscles with TPDN and K-Taping, with core muscle strengthening exercise protocol for 2 sessions with 3 days interval. He shows no symptoms of swelling, gait, numbness and tingling. However, decreased stability when trying to balance on right leg. Physiotherapist overall health was excellent and cleared from all red flags. Anti-inflammatory drug previously taken but no improvement in symptoms. The main aim for physiotherapy included decreased chronic low back pain.

\section{TREATMENT}

Patient was treated with dry needling along with K-taping for 2 sessions with three days interval. The $50 \mathrm{~mm}$ in length, $0.30 \mathrm{~mm}$ in diameter needle was used by the therapist. Firstly patient skin was inspected and disinfection was used with $70 \%$ isopropyl alcohol, needle was inserted using a clean method (fig.1,2).

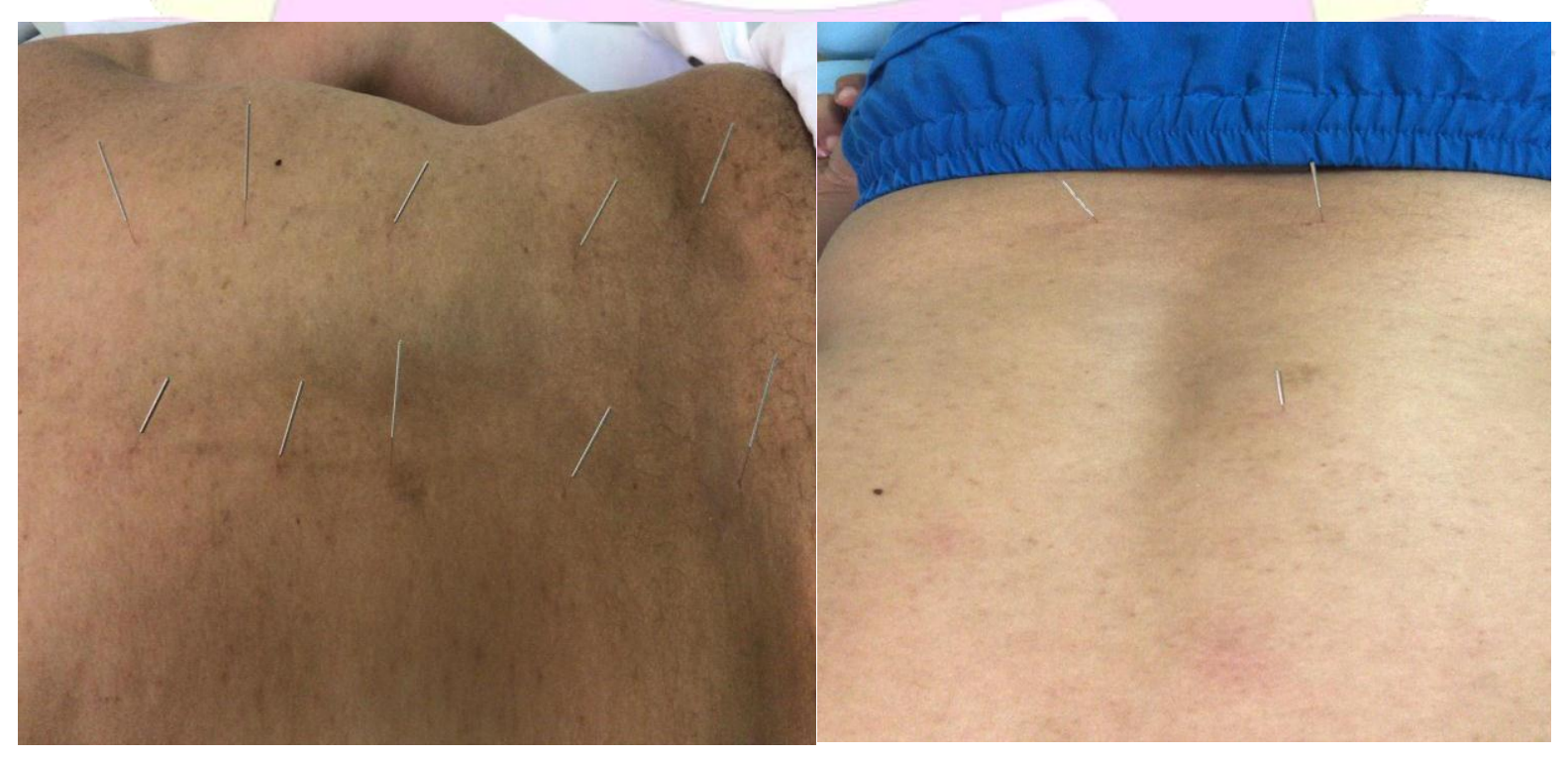


Figure 1- Figure. 2- $2^{\text {nd }}$ session with 3 dry needles Multifidus $1^{\text {st }}$ session of Dry needling technique. The patient was needle placements prone.

Five needles on each side of Para spinal was inserted into the lumbar multifidus muscle to the depth of the vertebral lamina.

Needle was pricked into the skin till the taut band over the Trigger Point (TP), then needle was directed into the target muscle TP. The needle was used in up and down manner (Pricking inside and taking out several time from TP) in a rapid frequency with a rate of approximately one- two strokes/sec. without taking full needle out from the skin. This "pistoning" provoking a local twitch response (LTR), by needle movement within a TP, and this attempt to reproduce the subject's symptoms. The needle was inserted many times to evoke as much as LTR local twitch responses is possible until the physiotherapist identify tissue changes. As soon as the needle was taken out from the skin, the pricked site was pressed gently for a minimum of $3 \mathrm{sec}$. and the needle was discarded into a yellow container. Patient was laid in prone position for multi fidus muscles, and the needle was inserted one finger breath just lateral to spinous process from midline. It was angled just medially to vertical axis and perpendicular to the lamina and inserted till its touching lamina, which was considered as a "bony backdrop" to make sure that needle is inserted in multifidus muscle. The index and middle fingers of the other hand was spread out to tension the skin. Following the TPDN treatment, the patient was given home advices and instructions to decrease post needling complications. He was also informed to take proper rest and keep hydrated for 24 hours and use ice pack on affected area if there is any muscle soreness. Two I shaped K-Tapes were applied from erector spine muscle from its origin to insertion in lumbar region. Treatment area was properly cleaned, hair free and measurement of K-tape was done with lumbar spine into full flexion. First four $\mathrm{cm}$ to five $\mathrm{cm}$ of K-tape was twisted and removed from its paper. The patient was asked to perform maximum flexion of spine, except for the final four $\mathrm{cm}$ to five $\mathrm{cm}$ and $\mathrm{K}$-tape was also used on one aspect paravertebrally within bone direction with mild traction. The ultimate four $\mathrm{cm}$ to five $\mathrm{cm}$ of $\mathrm{K}$ tape was applied without traction. Same method was used to on opposite side .Firm pressure was applied on K-tape by hand using repeated back and forth motion to warm the adhesive for proper adhesion (Figure 3$)^{21}$. 


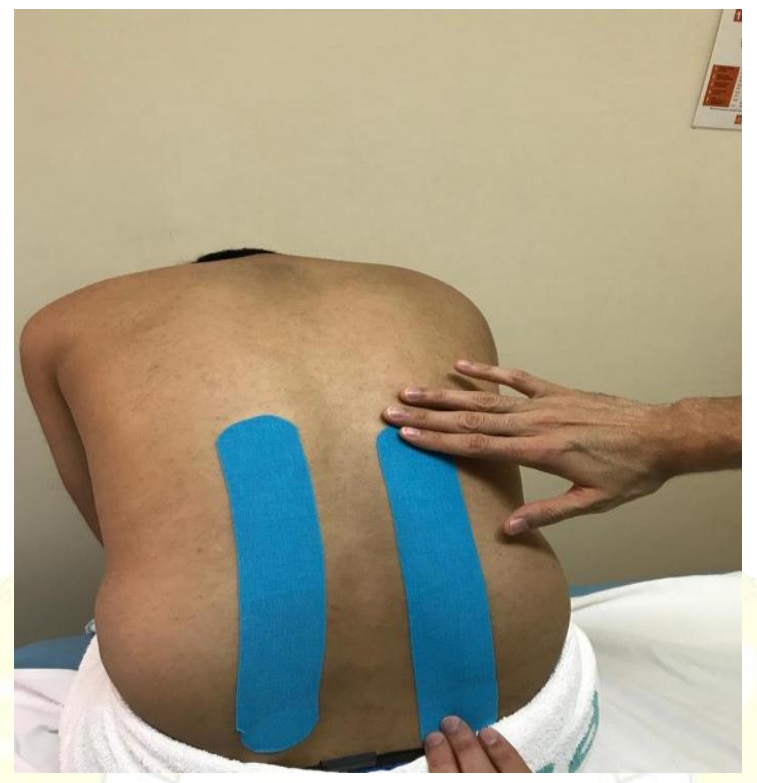

Figure 3- K-Taping Procedure

The patient was advised to perform core strengthening exercises twice daily as home exercise program (HEP). HEP on core stability mainly focused on flexion in supine and prone position $22-23$

\section{DISCUSSION}

The patient reported no pain in lumber region during activities of daily living in all directions. He was able to resume his previous job responsibilities without pain. There was significant reduction in pain after first $\mathrm{DN}$ session with $\mathrm{K}$-tape.

Shane L. Koppenhaver et. al (2015) was examined the possible associations between demographic, patient history, and physical examination variables and short-term clinical outcome following dry needling therapy performed on individuals with LBP. Six variables from participant demographics and the historical and physical examinations were associated with improvement in self-reported disability 1 week after dry needling treatment. Additionally, 2 of the variables (pain with the MLT and no aggravation with standing) demonstrated a multivariate relationship with improved disability ${ }^{24}$.

Charles E. (2013) reported that after dry needling treatment patient feel no pain in low back region during ADL like long sitting, bending, or lifting objects from ground. The patient was able to return to ADL and was able to perform job related duties. After two weeks patient returned to full duty without any restriction. Patient tolerated TPDN treatment without any post treatment side effects. Patient had decreased pain sensation post TPDN treatment and reported mild muscle soreness for two hours only localized to needle insertion point. Therefore, TPDN treatment along with IES can be used for the treatment of low back stability ${ }^{22}$.

Lin Liu et. al (2018) systematic review show the totality of evidence to date in relation to the effects of DN for the treatment of LBP and includes a total of 11 Randomized Control Trials 
on 802 subjects with LBP. The low-to-moderate-quality proof showed that in comparison to other treatment protocols, DN resulted in significant reduction in pain and disability at post intervention. But, DN along with other treatment methods for LBP were better than DN alone in decreasing pain at post intervention, but the quality of evidence was low ${ }^{5}$.

Ashraf Mahmoudzadeh et. al (2016) presented single-blind randomized clinical trial used to measure the effect of DN on pain intensity and functional disability in subjects with disco genic radiating LBP. The findings of the study confirm the hypothesis stating that DN improves pain and disability parameters in subjects treated with standard conservative physical therapy ${ }^{25}$. There is a scarcity of proof of efficaciousness of Kinesio tape in reducing low-back pain. Paoloni et al. found the impact of K-Tape along with exercise on pain and daily activities in subjects with Chronic Low Back Pain. They found statistically significant pain reduction on pain scale, at end of fourth week of intervention with K-Tape along with exercises ${ }^{26}$. Hwang Bo (2011) on his case study, found use of K-Tape on lumbar region for three days, decreased acute Low Back Pain and improved lumbar Range Of Motion. K-Tape can improve stability of joints, strength of weak muscles, decrease pain and correct posture ${ }^{18}$.

The physical property of Kinesio Tape will increase tension by stimulation throughout active trunk movement, which can facilitate pain gate mechanism through afferent fiber stimulation, hence decreasing pain sensation ${ }^{27}$.K-Tape enhance muscle action by improving strength of weak muscles ${ }^{16}$. All the above study strongly supports our result.

\section{REFERENCES}

1. Vos T, Flaxman AD, Naghavi M, et al. Years lived with disability (YLDs) for 1160 sequelae of 289 diseases and injuries 1990-2010: a systematic analysis for the Global Burden of Disease Study 2010. Lancet; 2012, 380:2163-96.

2. Han-Tong $\mathrm{Hu}$, et. al Is dry needling effective for low back pain? A systematic review and PRISMA-compliant meta-analysis, Medicine, 2018 97:26e11225.

3. Lim SS, Vos T, Flaxman AD, et al. A comparative risk assessment of burden of disease and injury attributable to 67 risk factors and risk factor clusters in 21 regions, 19902010: a systematic analysis for the Global Burden of Disease Study 2010. Lancet 2012;380: 2224 .

4. Maher C, Underwood M, Buchbinder R. Non-specific low back pain. Lancet 2017; 389:736-47.

5. Lin Liu et. al, Evidence for Dry Needling in the Management of Myofascial Trigger Points Associated With Low Back Pain: A Systematic Review and Meta-Analysis Archives of Physical Medicine and Rehabilitation 2018; 99:144-52. 
6. Deyo RA, Mirza SK, Martin BI. Back pain prevalence and visit rates: estimates from U.S. national surveys, 2002. Spine (Phila Pa 1976) 2006; 31:2724-7.

7. Van Middelkoop M, Rubinstein SM, Kuijpers T, et al. A systematic review on the effectiveness of physical and rehabilitation interventions for chronic non-specific low back pain. Eur Spine J 2011; 20:19-39.

8. Furlan AD, van Tulder MW, Cherkin DC, et al. Acupuncture and dry needling for low back pain. Cochrane Database Syst Rev 2005 ;( 1): CD001351.

9. Deyo RA, Von KM, Duhrkoop D. Opioids for low back pain. BMJ2015; 350:g6380.

10. Kalichman L, Vulfsons S. Dry needling in the management of musculoskeletal pain. J Am Board Fam Med 2010; 23:640-6.

11. Dunning J, Butts R, Mourad F, et al. Dry needling: a literature review with implications for clinical practice guidelines. Phys Ther Rev 2014; 19:252-65.

12. Liu L, Skinner MA, Mcdonough SM, et al. Traditional Chinese Medicine acupuncture and myofascial trigger needling: the same stimulation points? Complement Ther Med $2016 ; 26: 28-32$.

13. Yunus Atici et. al, The effect of Kinesio taping on back pain in patients with Lenke Type 1adolescent idiopathic scoliosis: A randomized controlled trial, Acta Orthopedic et Traumatologica Turcica 51, 2017, 191-196.

14. Kase K, Wallis J, Kase T. Clinical Therapeutic Applications of the Kinesio Taping Methods. Kinesio Taping Association; 2003.

15. Kenzo K. Elastic Therapeutic Taping: Let's Talk Treatment. Available from: http://www.dynamicchiropractic.com/mpacms/dc/article.php?id=55463. [Last cited on 2016 Oct 29].

16. Kim Trobec, Melita Peršolja, Efficacy of Kinesio Taping in reducing low back pain: A comprehensive review, Journal of Health Sciences 2017; 7(1):1-8.

17. West DJ, Gardner D. Occupational injuries of physiotherapists in North and Central Queensland. Aust J Physiother 2001; 47(3):179-83.

18. Gak Hwang Bo, Jung Hoon Lee, Effects Of Kinesio Taping In A Physical Therapist With Acute Low Back Pain Due To Patient Handling: A Case Report, International Journal of Occupational Medicine and Environmental Health 2011;24(3):320 - 323.

19. Glover W, McGregor A, Sullivan C, Hague J. Work-related musculoskeletal disorders affecting members of the Chartered Society of Physiotherapy. Physiotherapy 2005; 91(3):138-47. 
20. Darragh AR, Huddleston W, King P. Work-related musculoskeletal injuries and disorders among occupational and physical therapists. Am J Occup Ther 2009; 63(3):351-62.

21. Pijinappel, H., 2007. Handbook of Medical taping, concept. 1. Madrid: Aneid Press.

22. Charles E. Rainey, The Use Of Trigger Point Dry Needling And Intramuscular Electrical Stimulation For A Subject With Chronic Low Back Pain: A Case Report, IJSPT, 8, 2, 2013, 145-161.

23. Kleier DJ. Referred pain from a myofascial trigger point mimicking pain of endodontic origin. J Endod.1985; 11(9):408-411.

24. Shane L. Koppenhaver et. al, Baseline Examination Factors Associated With Clinical Improvement After Dry Needling in Individuals With Low Back Pain, JOSPT, 2015, 45,8,604-612.

25. Ashraf Mahmoudzadeh, Zahra Sadat Rezaeian, Abdolkarim Karimi, Jan Dommerholt, The effect of dry needling on the radiating pain in subjects with discogenic low-back pain: A randomized control trial, JRMS, DOI:10.4103/1735-1995.192502.

26. Paoloni, M., A Bernetti, G Fratocchi, M. Mangone, L. Parrilleno, M. Del Pilar, L. Cooper, Sesto, L. Di, Sante and V. Santilli, Kinesio taping applied to lumbar muscles influences clinical and electromyo graphic characteristics in chronic low back pain patients. Eur. J. Phys, Rehabil. Med. 2011, 47: 1-2.

27. Thelen MD, Dauber JA, Stoneman PD. The clinical efficacy of kinesio tape for shoulder pain: a randomized, double-blinded, clinical trial. J Orthop Sports Phys Ther 2008; 38(7):389-95.

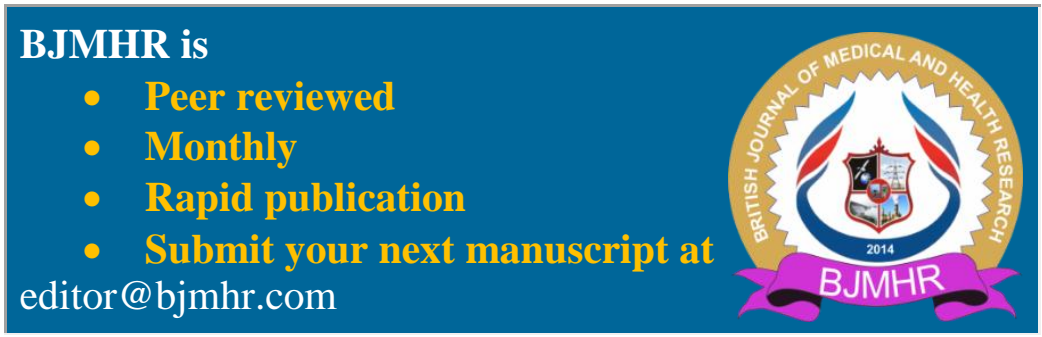

